Journal of Modern Optics, Vol. X, No. X, Month 2006, xxx-xxx

\title{
Parameterisation of an acousto-optic programmable dispersive filter for closed-loop learning experiments
}

\author{
NICHOLAS T FORM $\uparrow$, ROBERT BURBIDGE $\ddagger$, JAN RAMON AND BENJAMIN J WHITAKER $\dagger^{*}$ \\ $†$ School of Chemistry, University of Leeds, Leeds, LS2 9JT, UK \\ †Department of Computer Science, Penglais Campus, Aberystwyth, Ceredigion, SY23 3DB, Wales, UK
}

I Department of Computer Science, Katholieke Universiteit Leuven, Celestijnenlaan 200 A, B-3001 Heverlee, Belgium

*Corresponding author. Email: b.j.whitaker@leeds.ac.uk

\begin{abstract}
A representation of the search space in optical pulse shaping problems employing an acousto-optic programmable dispersive filter (AOPDF) is presented for use in closed-loop learning experiments where the optimal spectral phase function to some control problem is determined by an iterative learning algorithm. The representation allows the algorithm to select a value for the optical chirp at each frequency control point such that only acoustic grating functions which preserve the spectrum of the shaped pulses are tested. The limits of this space with respect to the rate of applied optical chirp, optical bandwidth and acoustic power are examined and tested through diffraction efficiency studies performed using a commercial AOPDF. The main benefits of this representation are the elimination of undesirable frequency mixing effects, reduction of diffraction efficiency variation between arbitrary pulse shapes and faster convergence of the evolutionary algorithm.
\end{abstract}

Keywords: Optical pulse shaping; Coherent Control; AOPDF; Closed-loop learning; Femtosecond laser

Words: 5472

\section{Introduction}

Although optimal control theory has long been applied to classical problems ${ }^{1}$ the application to quantum control problems is complicated by the general difficulty in knowing the system Hamiltonian to sufficient accuracy. This was imaginatively circumvented by Judson and Rabitz ${ }^{2}$ who in 1992 proposed a closed-loop strategy under the control of an evolutionary algorithm. The first practical demonstration ${ }^{3}$ of their scheme was performed in 1997 and the technique has since been applied to control a wide range of phenomena. Examples include pulse compression ${ }^{4}$, pulse propagation in non-linear media ${ }^{5}$, electronic population transfer ${ }^{3}$, high harmonic generation ${ }^{6}$ and the control of photofragmentation branching ratios ${ }^{7}$. Many of these studies have employed spatial light modulation (SLM) for optical pulse shaping, in which a liquid crystal element regularly divided into discrete pixels is placed at the Fourier plane of a 4-f zero-dispersion line. Because of the independence of the pixels in the liquid crystal array, SLMs are easily integrated into closed-loop setups where the voltages applied to each pixel are often adjusted directly by the learning algorithm with no need for intermediate translation into actual phase values ${ }^{7,8}$. 
The acousto-optic programmable dispersive filter (AOPDF) is a relatively new alternative to the $\mathrm{SLM}^{9}$. It consists of a birefringent crystal coupled to a radio-frequency (rf) pulse generator via a piezoelectric transducer to allow the introduction of a user programmable acoustic wave to the crystal. The crystal is placed directly in the beam path and oriented such that the light enters along the faster, ordinary optical axis. For lasers in the spectral range $0.5-4 \mu \mathrm{m}$ tellurium dioxide $\left(\mathrm{TeO}_{2}\right)$ is the most suitable material ${ }^{9}$ while recent results have shown that potassium dihydrogen phosphate (KDP) can be used at shorter wavelengths, from 500 down to $200 \mathrm{~nm}^{10}$. Shaping is achieved by means of the collinear acousto-optic interaction. This effect couples light between the ordinary and extraordinary modes of the crystal. Through appropriate design of the acoustic waveform, the relative distance travelled along the fast axis of the crystal prior to diffraction to the slow axis can be varied for each frequency component of the input optical pulse to achieve essentially arbitrary phase shaping.

The AOPDF is, like other optical pulse shaping devices, a spectral filter. However, unlike zerodispersion line techniques which employ transverse dispersion, AOPDFs use the longitudinal material dispersion of the crystal to separate the frequency spectrum for shaping. This feature offers several advantages in terms of installation and day-to-day use (for instance easy wavelength tuning) but also means that the phases of the individual optical frequency components become interdependent because of the need to diffract each frequency at a unique point along the length of the crystal with an acoustic pulse of finite intensity. The problem of identifying the acoustic phase function required to add an arbitrary optical phase $\phi(\omega)$ to the incoming electric field has already been addressed by Verluise et al. ${ }^{13}$ Their method is to first identify the position, $z(\omega)$, at which each frequency should be diffracted by considering the overall speed of each frequency component through the crystal. The necessary acoustic function is then calculated from the phase-matching condition required to diffract each frequency at the correct position. The authors also identified the range of optical phase functions that are available with an AOPDF in general but did not examine what, if any, further restrictions need to be placed on the programmed phase function in the case where one requires an invariant output optical spectrum $\left|E_{\text {out }}(\omega)\right|$.

It is obviously highly desirable in closed-loop pulse shaping experiments to maintain independent control of phase and amplitude. To achieve this we must design a parameterisation in which the spectral response of the shaper is invariant to the programmed phase function, while at the same time retaining the widest possible range of phase functions in line with the conjecture of Rabitz et al. that in evolutionary optimization experiments one should place as few restrictions on the control field as possible ${ }^{14}$. The essence of the problem is that the amplitude and phase shaping capabilities of an AOPDF are intimately coupled and are only separable under certain conditions. In fact, this coupling is also a "feature" of SLM and deformable mirror phase shapers, albeit for different reasons. Coupling can lead to undesired spectral amplitude modulation and means that one cannot apply completely arbitrary phase functions to the device and expect them to be faithfully reproduced in the optical field. That is there are physical limitations on the acoustic waveforms that can be transferred to the optical pulse. We will explore the nature of these constraints more fully below but for the moment note that previously successful examples of closed-loop experiments with AOPDFs have employed several different parameterisations of the spectral phase. An obvious method is to use the coefficients of a Taylor expansion of the phase function made around the central frequency, as reported by Ohno et al. ${ }^{15}$ Taylor expanded spectral phase functions are particularly effective ways of describing material dispersion and for this reason are useful in AOPDF applications where the dispersion introduced by the crystal itself must be accounted for. It is fairly easy to identify approximate ranges for the linear and quadratic coefficients that ensure that the acoustic phase is copied to the optical phase but is increasingly difficult to so do for higher order terms. On the other hand, truncating the series at a low order will exclude many valid potential pulse shapes from the searchable space. Another previously reported 
parameterisation method used the modulo $2 \pi$ values of the phase for a subset (10) of the $N$ frequency control points across the shapable bandwidth ${ }^{16}$. Although optimisations using this parameterisation were successful; in this case in the goal of increasing high harmonic generation; it is difficult to represent the physical constraints on the realizable phase functions effectively in this space. While it would be possible to simply reject offending pulses, this is computationally inefficient and a space in which such pulses are automatically excluded is preferred. Our aim here is to design such a parameterisation and to demonstrate that it is compliant with the physical constraints on the realizable phase functions we will identify.

\section{Parameterisation of the search space}

The operation performed by the AOPDF is described as the convolution of the acoustic waveform in scaled time, $S(t / \alpha(\omega))$, with the input optical field $E_{\text {in }}(t)$ :

$$
E_{\text {out }}(t) \propto E_{\text {in }}(t) \otimes S(t / \alpha(\omega)),
$$

where $\alpha(\omega)$ is a frequency dependent scaling factor accounting for the speed ratio of light and sound in the crystal $^{11}$. The creation of an arbitrary $S(t)$ within the crystal at the instant of the arrival of the optical pulse will transfer the corresponding phase function onto $E_{\text {in }}(t)$ to give an arbitrary pulse shape in the output electrical field, $E_{\text {out }}(t)$.

It is convenient to rewrite eqn. (1) in terms of the spectrum:

$$
E_{\text {out }}(\omega) \propto E_{\text {in }}(\omega) A(\Omega) \exp [i \Phi(\Omega)],
$$

where the acoustic frequency is given by:

$$
\Omega=\omega \cdot \alpha(\omega),
$$

and $A(\Omega)$ and $\Phi(\Omega)$ are real functions describing the amplitude and phase of the Fourier transform of $S(t)$ respectively. It can be shown that, to a first approximation, the amplitude, $\left|E_{\text {out }}(\omega)\right|$, and additional phase, $\phi(\omega)$, of the output optical pulse can be controlled independently through $A(\Omega)$ and $\Phi(\Omega)^{9}$. Thus, $S(t)$ is programmed as the real part of the inverse Fourier transform of the complex spectrum created by $A(\Omega)$ and $\exp [i \Phi(\Omega)]$ specified at the set of points defined by the spectral resolution of the shaper. For an optical bandwidth of $\Delta \lambda$ this requires a list of $N$ amplitudes and phases where:

$$
N=\frac{\Delta n\left(\lambda_{0}\right)}{0.8} \cdot L \cos ^{2} \theta_{[110]} \cdot \frac{\Delta \lambda}{\lambda_{0}^{2}},
$$

for a pulse with central wavelength $\lambda_{0}$ propagating in the P-plane at angle $\theta_{[110]}$ to the [110] axis (in the case of $\mathrm{TeO}_{2}$ ) of the AOPDF crystal of length $L$ and birefringence $\Delta n^{12}$. Here $\Delta \lambda$ is simply the difference between the long and short wavelength cut-offs of the desired diffracted bandwidth in a square window function. For $\Delta \lambda=42 \mathrm{~nm}, \lambda_{0}=540 \mathrm{~nm}, \theta_{[110]}=45^{\circ}$ and $L=25 \mathrm{~mm}, N$ is 359 . Another useful quantity is the maximum programmable delay ${ }^{12}$ :

$$
\tau_{\max }=\Delta n_{g}\left(\lambda_{0}\right) \cos ^{2}\left(\theta_{[110]}\right) L / c,
$$

where $\Delta n_{g}\left(\lambda_{0}\right)$ is the group birefringence at the central wavelength. For the conditions stated, $\tau_{\max }=8.75 \mathrm{ps}$.

The amplitude and phase shaping capabilities of an AOPDF are only separable, as implied by eqn. (2), for phase functions obeying the following constraints:

1. Each optical frequency must be diffracted at a unique position.

2. Each optical frequency must be diffracted within the length, $L$, of the crystal, or equivalently within a delay range between 0 and $\tau_{\max }$. 
3. The intensity of the acoustic waveform, $S^{2}(t)$, must not exceed the maximum intensity, $I_{\max }$, that can be introduced into the crystal without distortion by the electronics of the rf generator.

The first condition prevents mixing of optical frequencies that would result from superposing their diffraction at the same point in the crystal. This constraint was implicit in the analysis of Verluise et al. as was the second which ensures that all the available bandwidth is transferred to the diffracted beam. The final constraint identified here ensures hi-fidelity of the acoustic pulse, a pre-requisite for accurate pulse shaping. While violation of the first two constraints can occur no matter what the acoustic energy, violation of the third only occurs for acoustic pulses above a certain intensity threshold. Since the efficiency with which the optical pulse is diffracted is proportional to the acoustic energy, the restriction imposed by the acoustic intensity limit under normal working conditions is important experimentally. In principle, the power and peak intensity of the acoustic wave is fundamentally limited by the threshold above which heat and/or mechanical damage occurs in the crystal. Experimentally, however, it turns out that amplifier distortion is of greater importance.

Since constant and linear terms in the spectral phase do not affect the shape of a pulse, operating directly on the phases of the frequency control points leads to an infinite redundancy in the search space. To remove this we shall assume the constant delay term to be fixed as $\tau_{\max } / 2$ so as to centre the acoustic pulse in the crystal at the instant the optical pulse arrives. Note that although the acoustic pulse propagates through the crystal the respective magnitudes of the speed of light and sound in the crystal mean that the acoustic wave is effectively stationary during its interaction with the optical pulse. Experimentally the correct delay is achieved by triggering the rf generator a few micro seconds in advance of the light pulse. In what follows we shall make use of the group delay (or simply delay) and group delay dispersion (also known as linear chirp) of the pulse, defined as the first and second derivatives respectively of the spectral phase. We shall use primes to denote differentiation with respect to frequency and hence denote the optical delay added to the pulse as $\phi^{\prime}(\omega)$ and the corresponding dispersion as $\phi^{\prime \prime}(\omega)$. Also, where we wish to denote a term as constant with respect to frequency, we shall simply omit the bracketed frequency symbol.

An alternative method for identifying $\Phi(\Omega)$ to that described by Verluise et al. (op. cit.) is to identify the programmed dispersion function $\phi_{\text {prog }}^{\prime \prime}(\omega)$ corresponding to $\phi^{\prime \prime}(\omega)$ as:

$$
\phi_{\text {prog }}^{\prime \prime}(\omega)=\phi^{\prime \prime}(\omega)-\phi_{\text {crystal }}^{\prime \prime}(\omega) \text {, }
$$

where $\phi_{\text {crystal }}^{\prime \prime}(\omega)$ represents the dispersion resulting from propagation half way along the ordinary and half way along the extraordinary axis of the crystal and $\phi^{\prime \prime}(\omega)$ is the desired optical dispersion to be added to the input pulse. To identify the corresponding acoustic phase function we integrate $\phi_{\text {prog }}^{\prime \prime}(\omega)$ twice (with $\tau_{\max } / 2$ as the first constant of integration) to give the programmed phase function and then simply assign:

$$
\Phi(\Omega)=\phi_{\text {prog }}(\omega)
$$

where eqn. (3) gives the relationship between optical and acoustic frequencies. Limiting the delay function, $\phi_{\text {prog }}^{\prime}(\omega)$, to the delay range $0 \ldots \tau_{\max }$ satisfies constraint 2 . To avoid frequency mixing, we must impose the further restriction that $\phi_{\text {prog }}^{\prime}(\omega)$ be strictly monotonic (either increasing or decreasing) within the stated range. This now leads to an upper bound for the absolute value of the dispersion that can be programmed of:

$$
\left|\phi_{\text {prog }}^{\prime \prime}\right|_{\max }=\tau_{\max } / \Delta \omega,
$$

where $\Delta \omega$ is the optical frequency bandwidth corresponding to the same long and short wavelength cut-offs as $\Delta \lambda$. Eqn. 8 represents the average value of the maximum programmable chirp since we are simply defining the absolute limit on the applied linear chirp as the maximum delay divided by the frequency bandwidth over the entire crystal. Locally slightly higher chirp values could be achieved at points within the 
crystal without causing the acoustic waveform to extend outside the crystal provided that these high chirp rates where compensated for elsewhere in the crystal. The constraint implied by eqn. (8) is, however, computationally convenient and in practice not over restrictive.

We can also identify a lower limit for the absolute programmed dispersion, $\left|\phi_{\text {prog }}^{\prime \prime}\right|_{\min }$ by noting that $\left|\phi_{\text {prog }}^{\prime \prime}\right|$ is approximately proportional to the acoustic linear chirp $\left|\Phi^{\prime \prime}\right|$. Despite eqn. (7), the correspondence is not exact because of the frequency dependence of the velocity ratio $\alpha$ (see eqn. (3)), but the important point is that the peak intensity of the pulse will vary with the dispersion. For a Gaussian pulse, one can easily show that for isoenergetic pulses the intensity should be proportional to

$$
I_{\text {peak }} \propto\left(\sqrt{1+\left(\frac{\phi^{\prime \prime} \ln 2}{\delta t^{2}}\right)^{2}}\right)^{-1},
$$

where $\delta t$ is the FWHM pulse width. Thus, because there is a physical limit to the acoustic power that can be applied to the crystal (either a result of an imperfect amplifier or ultimately because of material damage) there is a minimum limit for the absolute programmable dispersion. Attempts to programme values below this threshold will require too intense an acoustic pulse and cannot be faithfully reproduced. Such acoustic waveforms will result in an amplitude distortion of the diffracted optical pulse.

The restriction imposed on the phase function by the acoustic intensity limit depends on the acoustic spectrum because a high acoustic pulse energy requires a large chirp in order to avoid intensities in excess of $I_{\max }$. Clearly, for phase only shaping the magnitude of the acoustic spectrum, $A(\Omega)$, must remain independent of $\Phi(\Omega)$. This can be achieved by fixing the shape of the acoustic spectrum and then scaling the intensity of the acoustic waveform appropriately. In practice, this is done by loading an initial waveform and scaling all subsequent pulses to contain the same total acoustic power and hence give the same overall diffraction efficiency. If we were to choose the acoustic waveform which exactly compensates the linear dispersion of the crystal then the programmed optical delay $\phi_{\text {prog }}^{\prime \prime}$ must equal $-\phi_{\text {crystal }}^{\prime \prime}$. If this is scaled to a peak intensity of $I_{\max }$ then we would have to set $\left|\phi_{\text {prog }}^{\prime \prime}\right|_{\min }=\left|\phi_{\text {crystal }}^{\prime \prime}\right|$ since pulses with lower absolute linear chirps would immediately exceed the maximum allowable acoustic intensity. If instead we set the acoustic pulse energy to be that of the self-compensating waveform scaled to a peak intensity $I \cdot I_{\max }$, where $I$ is a number between 0 and 1, we would decrease the lower bound to approximately:

$$
\left|\phi_{\text {prog }}^{\prime \prime}\right|_{\text {min }}=\frac{\sqrt{I^{2} \delta t^{4}+\left(I\left|\phi_{\text {crystal }}^{\prime \prime}\right| \ln 2\right)^{2}-\delta t^{4}}}{\ln 2},
$$

which allows us to trade diffraction efficiency against shaping capability. Eqn. (10) is obtained by substituting $\left|\phi_{\text {crystal }}^{\prime \prime}\right|$ into (9), dividing by $I$ and finding the value of $\phi_{\text {prog }}^{\prime \prime}$ which gives the same overall intensity.

Although we have already discussed how we can meet the need to diffract each frequency component of the optical pulse at a unique depth within the crystal by ensuring that the programmed delay function is strictly monotonic this condition alone is not sufficient to avoid frequency mixing. Specifically, there is a lower limit for the absolute linear chirp associated with these non-linear optical processes that is separate from that expressed in eqn. (10). This can be thought of as a minimum distance between the diffraction positions of neighbouring frequency components. We will not derive an eqn. for this lower limit but will instead show experimentally that it is much less restrictive than that expressed in eqn. (10) for all but the lowest diffraction efficiencies. Nevertheless, unlike amplifier distortion which may be avoided altogether when the intensity of 
$A(\Omega)$ is small enough, optical frequency mixing can occur for any acoustic spectrum for a programmed chirp close to zero.

Until now we have assumed the programmed chirp to be constant with respect to frequency but having identified the range in which it can vary we may now let $\left|\phi_{\text {prog }}^{\prime \prime}(\omega)\right|$ become a function of frequency, thus introducing the possibility for higher order phase variation. By configuring the learning algorithm to operate on the $N$ values of $\left|\phi_{\text {prog }}^{\prime \prime}(\omega)\right|$ in the range $\left|\phi_{\text {prog }}^{\prime \prime}\right|_{\min } \ldots\left|\phi_{\text {prog }}^{\prime \prime}\right|_{\max }$, as well as one further binary variable determining the overall sign of the function, $s= \pm 1$, we enable the search algorithm to select only functions with a constant spectral response. The additional phase added to the optical pulse by the shaper is calculated by solving eqn. (6) for $\phi^{\prime \prime}(\omega)$ and integrating twice as described. This parameterisation allows a learning algorithm to select the additional delay at each frequency control point as depicted in Figure 1. At the first point in the frequency list $\left\{\omega_{i}\right\}$ a delay lying in the range indicated by the dashed lines is chosen randomly. At each subsequent frequency in the list an additional delay can be chosen at random provided that the incremental rate of change of the delay does not exceed $\left|\phi_{\text {prog }}^{\prime \prime}\right|_{\max }$ or become less than $\left|\phi_{\text {prog }}^{\prime \prime}\right|_{\min }$. The overall delay at each frequency control point is thus the delay accumulated over the preceding portion of the spectrum. This procedure ensures that the programmed delay $\phi_{\text {prog }}^{\prime}(\omega)$ is a strictly monotonic function (the sign depending on a random flip of a coin.)

Two aspects of this parameterisation methodology are worthy of further comment. Firstly, it follows from eqn. (6) that the symmetric limits identified for the programmed linear chirp, $\phi_{\text {prog }}^{\prime \prime}$, correspond to asymmetric limits for the additional linear optical chirp, $\phi^{\prime \prime}$. This leads to the situation shown in Figure 1 whereby the linear component of the self-compensation function (dotted line) lies close to the maximum negative programmable dispersion. The separation between the self-compensation function and the limit of the space can be increased by reducing the bandwidth, $\Delta \omega$, or by reducing the crystal dispersion, $\phi_{\text {crystal }}^{\prime \prime}$. The latter can be achieved using a grating or prism compressor to pre-compensate for the dispersion of the AOPDF. In the context of the application of our parameterization scheme to a learning algorithm the choice of -1 for the sign of $\phi_{\text {prog }}^{\prime \prime}(\omega)$ is generally the more useful since then the programmable or additional phase function $\phi(\omega)$ can contain both positive and negative chirp terms with respect to the input phase. Although pulses selected from the oppositely signed portion of the allowable delay space should have the same diffraction efficiency and are in principle perfectly good potential candidate solutions. Secondly, it should be noted that the limits identified in eqns. (8) and (10) are slightly over-restrictive on the allowable programmed dispersion as they are calculated on the basis of linear chirp only. As we have pointed out, the upper bound is calculated by dividing the available programmable delay range by the bandwidth. This implies assigning a roughly equal portion of the delay to each wavelength control point (roughly equal because the spectral resolution varies as a function of wavelength). If one were to program a low value of $\left|\phi_{\text {prog }}^{\prime \prime}(\omega)\right|$ at one frequency however then more delay would become available at later frequencies in the control list. Similarly, a chirp lower than $\left|\phi_{\text {prog }}^{\prime \prime}\right|_{\text {min }}$ at just one control point will not immediately lead to intensities above $I_{\max }$. It is possible to conceive of various strategies to slightly decrease the restriction imposed by $\left|\phi_{\text {prog }}^{\prime \prime}\right|_{\min }$ and $\left|\phi_{\text {prog }}^{\prime \prime}\right|_{\max }$ but at the cost of a considerable computational overhead. 


\section{Experimental}

Pulses centred at $540 \mathrm{~nm}$ from a commercial non-collinear optical parametric amplifier (Clark-MXR, NOPA) pumped by a $1 \mathrm{kHz}$ repetition chirped pulse amplifier (Clark-MXR, CPA-2010) are directed into an AOPDF for visible frequency pulse shaping (Fastlite, Dazzler). The AOPDF is a $\mathrm{TeO}_{2}$ crystal of length $L=25 \mathrm{~mm}$ for which the propagation angle $\theta_{[110]}=45^{\circ}$. The pulse energy directed into the AOPDF is approximately $3 \mu \mathrm{J}$. The maximum spectral shaping bandwidth of the AOPDF (42 nm at this wavelength) is employed throughout leading to an upper absolute chirp limit of $\left|\phi_{\operatorname{prog}}^{\prime \prime}\right|_{\max }=32039 \mathrm{fs}^{2}$ (from eqn. 8)

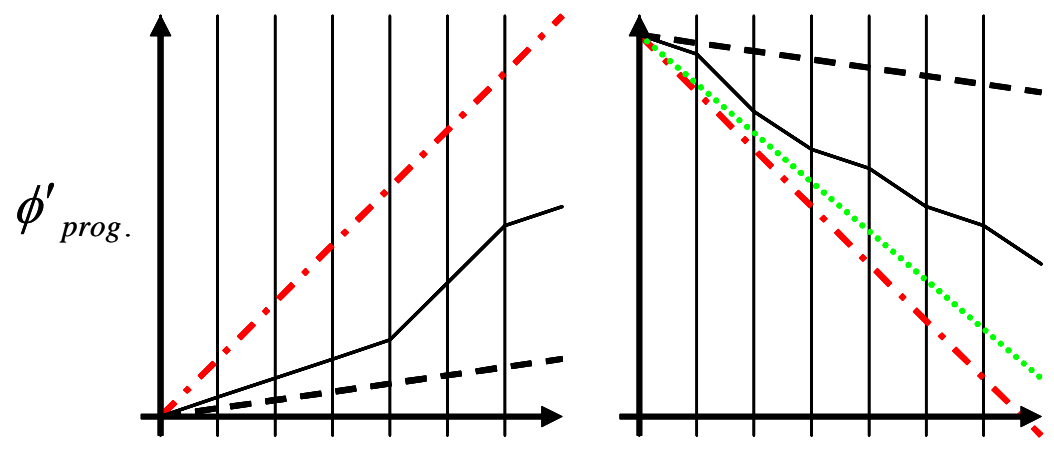

$\omega$

Figure 1 - Pictorial representation of the programmed delay space for $s=1$ (left) and -1 (right), where $s$ is the sign of the programmed chirp function (see text for details). The vertical lines represent the frequency control points. The maximum (dot-dashed line) and minimum (dashed line) chirp rates are shown for each case along with an example of an allowable programmed delay function (solid line). The dotted line depicted in the -1 case represents the delay function required to compensate the linear dispersion of the crystal.

To measure the spectrally resolved diffraction efficiency the undiffracted light, propagating along the ordinary axis of the crystal, is collected by a $\sim 4 \pi$ integrating sphere which is fibre-coupled to a diode array spectrometer (Ocean Optics, FOIS-1 and HR4000). Firstly, a spectrum of the light emerging on this mode is taken with no acoustic wave present. Next, acoustic waveforms with $\pm\left|\phi_{\text {prog }}^{\prime \prime}\right|_{\max }$ are loaded in turn and spectra averaged over several thousand laser shots recorded. This process was repeated at least three times under each set of conditions and the spectra for each case averaged before being combined as one minus the intensity ratio of the undiffracted beam with and without the acoustic wave present to give the AOPDF spectral response.

We then measured the diffracted beam spectrum, propagating along the extraordinary axis, as a function of $\phi_{\text {prog }}^{\prime \prime}$. To observe the effects of optical frequency mixing processes we loaded a transform limited acoustic pulse to set the maximum intensity. In this way we remove the lower chirp limit with respect to constraint 3 altogether, but at the expense of overall diffraction efficiency since under these conditions the total acoustic power that can be coupled into the crystal is modest. To demonstrate the effects of amplifier distortion under conditions giving higher diffraction efficiency we set $I=0.48$ for the self compensation waveform $\left(\phi_{\text {crystal }}^{\prime \prime}=27557 \mathrm{fs}^{2}\right)$, giving a lower absolute chirp limit of $\left|\phi_{\text {prog }}^{\prime \prime}\right|_{\min }=13179 \mathrm{fs}^{2}$ with $\delta t=30 \mathrm{fs}$. 


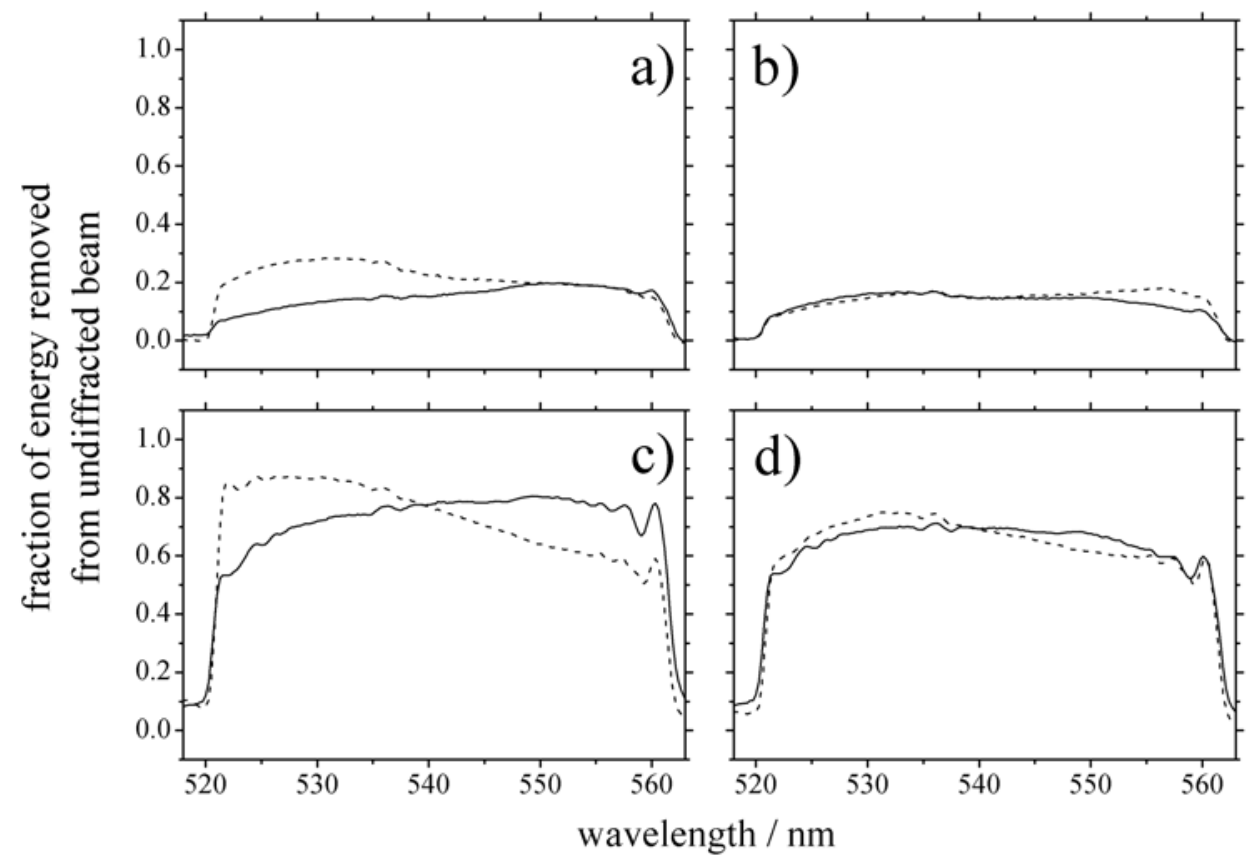

Figure 2 - Spectrally resolved diffraction efficiencies for $+\left|\phi_{\mathrm{prog}}^{\prime \prime}\right|_{\max }$ (dashed curves) and $-\left|\phi_{\text {prog }}^{\prime \prime}\right|_{\max }$ (solid curves) under various different conditions; a) $I=0.1$, no depth correction $\mathbf{b}$ ) $I=$ 0.1 , with depth correction c) $I=1.0$, no depth correction d) $I=1.0$, with depth correction.

\section{Results and discussion}

So far we have ignored the issue of acoustic attenuation. This reduces the coupling efficiency between the ordinary and extraordinary axes as the acoustic pulse travels further into the crystal. This problem becomes more serious at high acoustic frequencies and is the principal design constraint on building AOPDFs for short wavelengths ${ }^{17}$. In the presence of linear chirp this artefact can also have an undesired amplitude shaping effect and must be corrected for. This is achieved by applying a depth-dependent correction function to the acoustic waveform. The correction function can be obtained empirically as $C(z / L)=D(z / L)^{1 / 2}$ where $D(z / L)$ is the measured diffraction efficiency of a short acoustic pulse as a function of the depth into the crystal, $z$. This is simply done by scanning the short acoustic pulse (typically chirped to $-1000 \mathrm{fs}^{2}$ to avoid frequency mixing effects around $\phi_{\text {prog }}^{\prime \prime}=0$ ) through the available delay range from 0 to $\tau_{\text {max }}$. Alternatively, an empirical algebraic expression may be employed. At $540 \mathrm{~nm}$ we have found the function $C(z / L)=0.545+$ $0.32(z / L)+0.165(z / L)^{2}$ to give a diffraction efficiency flat to better than $5 \%$ across the central $80 \%$ of the crystal by scaling the acoustic intensity at a relative depth $z / \mathrm{L}$ at the instant of arrival of the laser pulse by $1 / C(z / L)$.

The significant improvement made by this correction can be seen by comparing the diffraction efficiency spectra in panels a) and c) (correction disabled) of Figure 2 with those in panels b) and d) (correction enabled) respectively. The two curves in each panel represent the positive (dashed) and negative 
(solid) acoustic linear chirps that completely fill the crystal in accordance with eqn. 8 . The correspondence between the up- and down-chirped curves can be seen to be best in the case of low acoustic power. The residual differences between the two corrected curves arise from the wavelength dependence of the correction factor, which we have ignored here but which could be added in a relatively straightforward manner. As we have mentioned, the acoustic attenuation is most severe at high frequencies and the data presented here are close to a worst-case scenario for $\mathrm{TeO}_{2}$ as they employ the maximum bandwidth at a central wavelength approaching the short-wavelength cut-off of this material.

With the acoustic attenuation correction function in place we measured the diffracted spectrum as a function of $\phi_{\mathrm{prog}}^{\prime \prime}$. Figure 3 depicts the results for the two settings of the acoustic spectral amplitude function $A(\Omega)$ described in Section 3. The upper and lower bounds on $\left|\phi_{\text {prog }}^{\prime \prime}\right|$ calculated using eqns. (8) and (10) are indicated by the vertical dashed lines and are seen to be appropriate in both cases. By comparing the two halves of the figure it can be seen that, under the conditions chosen here, the effects of optical frequency mixing processes (Figure 3a) are much more localised around the zero of the programmed linear chirp than the effects of amplifier distortion (Figure 3b). This confirms that under normal operating conditions the

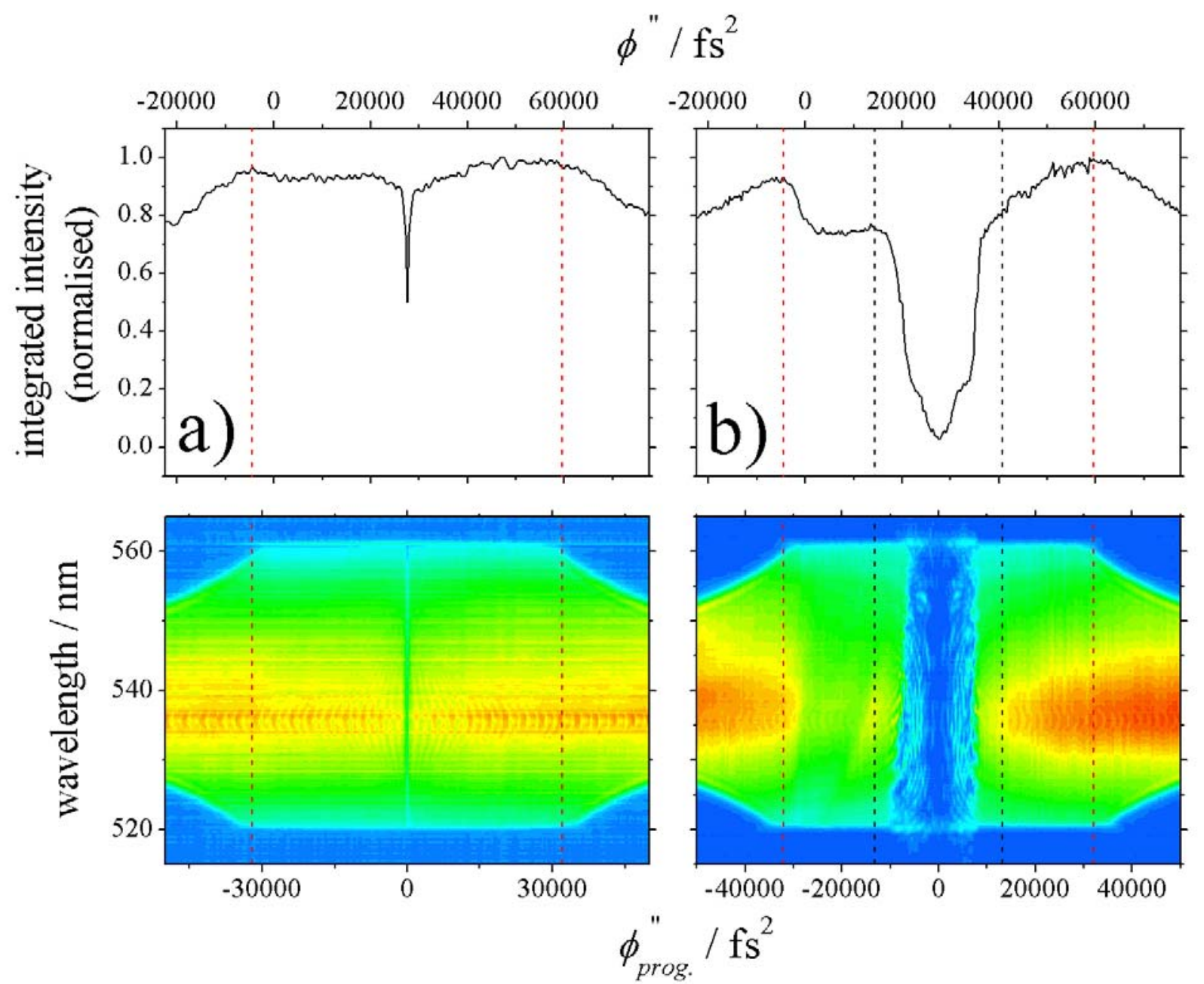

Figure 3 - Diffracted $540 \mathrm{~nm}$ spectrum (bottom) and normalised integrated intensity (top) as a function of $\phi_{\text {prog }}^{\prime \prime}$ (bottom axis) and $\phi^{\prime \prime}$ (top axis) for two settings of $A(\Omega)$. Dashed vertical lines indicate the calculated limits for absolute programmed linear chirp. From a) non-linear optical effects can be seen to play a significant role only in the immediacy of the acoustic transform limit, while b) demonstrates that distortion of the r. ${ }^{-}$enerator spans a far wider range for a setting of $I=0.48$. 
requirement to limit the intensity of the acoustic waveform is more restrictive than the requirement to diffract each frequency component at a different depth within the crystal.

It is worthwhile to reiterate that the space represented in this parameterisation is asymmetric with respect to the total added chirp due to the dispersion of the crystal. This is illustrated in Figure 3 by the topmost axis. As mentioned, the asymmetry between these ranges can be reduced with the addition of a grating or prism compressor adding negative linear chirp to effectively reduce the $\phi_{\text {crystal }}^{\prime \prime}$ term although this will lead to lower diffraction efficiency for the same setting of the acoustic power. Despite the reduced efficiency, the alignment difficulties associated with an SLM are still likely to be greater than those arising from a combination of a compressor with an AOPDF because the compressor in this case does not need to be as well aligned as in an SLM (because third and higher order dispersion effects due to slight misalignment of the compressor can be easily compensated for in the AOPDF). It is also possible to adjust values of $\left|\phi_{\text {prog }}^{\prime \prime}\right|_{\text {min }}$ and $\left|\phi_{\text {prog }}^{\prime \prime}\right|_{\max }$ since the parameters $I$ and $\Delta \omega$ may be tailored to the experiment to give a particular combination of power, bandwidth and shaping capability. Although we have shown that one can obtain decoupling of phase and amplitude at high diffraction efficiency over a restricted range of phase functions, this does not mean that the AOPDF is not suitable for closed-loop control applications (or indeed that it is an inferior device to say an SLM, which suffers from a different but related problem originating in the pixelation). Figure $3 \mathrm{~b}$ shows that for $I=0.48$ corresponding to a diffraction efficiency slightly above $40 \%$, an additional chirp in the range $-4482 \mathrm{fs}^{2}$ to $14378 \mathrm{fs}^{2}$ or 40736 to $59589 \mathrm{fs}^{2}$ is available to a search algorithm (depending on the sign chosen for $\phi_{\text {prog }}^{\prime \prime}$ ). Such rates are significant in, for example, molecular control problems. For example as recently demonstrated by Prokhorenko et al. who used an AOPDF in a closed-loop optimisation of retinal isomerisation in bacteriorhodopsin. ${ }^{18}$

\section{Conclusion}

A representation of the true arbitrary phase-only shaping capability of an AOPDF has been devised. We have investigated the range of programmed chirp for which spectrally flat diffraction efficiency can be expected. Experimental data have been presented to support these limits. Arbitrary pulses generated by selecting an instantaneous linear chirp from this space for each frequency control point thus represent the most complete set of optical pulse shapes that can be created with an AOPDF without spectral distortion (in fact, a slightly less restrictive space can be generated, but at additional computational expense). The corresponding phase functions can be obtained by adding the material dispersion to the selected values and twice integrating the resulting function numerically with respect to frequency. This representation of the possible pulse shapes is most useful in closed-loop learning applications such as coherent control. In these applications, one typically selects a small population of randomly generated pulse shapes, applies them to the control problem and then uses evolutionary pressure to select a small sub-set of best or "fittest" pulses to seed the next generation. The evolutionary algorithm then optimises the control pulse from generation to generation by maximising its fitness. In this representation, the instantaneous linear chirp at each frequency control point can be regarded as a gene in the chromosome defining the pulse. By confining the genes to the range of chirp we have identified one is able to restrict the learning algorithm to explore only those regions of the optical phase space which do not also result in unintended spectral modulation. This prevents the evolutionary algorithm from improving the fitness through trivial intensity effects. 
Journal of Modern Optics, Vol. X, No. X, Month 2006, xxx-xxx

\section{Acknowledgements}

This work has been supported by EPSRC grant S47649. NTF also thanks EPSRC for the award of a research studentship. We are also grateful to Prof Ross King and Dr Jem Rowland (Department of Computer Science, Aberystwyth, Wales) and Dr Daniel Kaplan (Fastlite, Palaiseau, France) for stimulating discussions. 


\section{References}

L. S. Pontryagin, V. G. Boltyanskii, R. V. Gamkrelidze, and E. F. Mishchenko, The Mathematical Theory of Optimal Processes. (John Wiley \& Sons, Inc.. New York. , 1962).

R. S. Judson and H. Rabitz, Phys. Rev. Lett., 68, 1500 (1992).

C. J. Bardeen, V. V. Yakovlev, K. R. Wilson, S. D. Carpenter, P. M. Weber, and W. S. Warren, Chem. Phys. Lett., 280, 151 (1997).

T. Baumert, T. Brixner, V. Seyfried, M. Strehle, and G. Gerber, Appl. Phys. B, 65, 779 (1997).

F. G. Omenetto, J. A. Taylor, M. D. Moores, and D. H. Reitze, Opt. Lett., 26 (12), 938 (2001).

R. Bartels, S. Backus, E. Zeek, L. Misoguti, G. Vdovin, I. P. Christov, M. M. Murnane, and H. C. Kapteyn, Nature, 406, 164 (2000).

A. Assion, T. Baumert, M. Bergt, T. Brixner, B. Kiefer, V. Seyfried, M. Strehle, and G. Gerber,

Science 282, 919 (1998); R. J. Levis, G. M. Menkir, and H. Rabitz, Science, 292, 709 (2001).

D. Zeidler, S. Frey, K. Kompa, and M. Motzkus, Phys. Rev. A, 64, 023420 (2001).

P. Tournois, Opt. Comm., 140, 245 (1997).

S. Coudreau, D. Kaplan, and P. Tournois, Optics Letters, 31, 1899 (2006).

F. Verluise, V. Laude, Z. Cheng, C. Spielmann, and P. Tournois, Optics Letters, 25, 575 (2000).

D. Kaplan and P. Tournois, Journal de Physique IV, 12, 69 (2002).

F. Verluise, V. Laude, J.-P. Huignard, P. Tournois, and A. Migus, Journal of the Optical Society of America B: Optical Physics, 17, 138 (2000).

H. A. Rabitz, M. M. Hsieh, and C. M. Rosenthal, Science, 303 (5666), 1998 (2004).

K. Ohno, T. Tanabe, and F. Kannari, Journal of the Optical Society of America B: Optical Physics, 19, 2781 (2002).

D. H. Reitze, S. Kazamias, F. Weihe, G. Mullot, D. Douillet, F. Auge, O. Albert, V. Ramanathan, J. P. Chambaret, D. Hulin, and P. Balcou, Optics Letters, 29, 86 (2004).

A. Monmayrant, A. Arbouet, B. Girard, B. Chatel, A. Barman, B. J. Whitaker, and D. Kaplan, Applied Physics B: Lasers and Optics, 81, 177 (2005).

V. I. Prokhorenko, A. M. Nagy, S. A. Waschuk, L. S. Brown, R. R. Birge, and R. J. D. Miller, Science, 313, 1257 (2006). 